\title{
On soluble groups of prime-power exponent
}

\section{Warren Brisley and L. G. Kovács}

\author{
Let $p$ be a prime and $\frac{A^{2}}{p}$ the variety of elementary abelian \\ by elementary abelian p-groups. A result of Brisley and \\ Macdonald is generalized as follows. If $H$ is a finite group \\ in $\stackrel{A_{p}^{2}}{=}$ and $G$ is a soluble group of $p$-power exponent such \\ that no section of $G$ is isomorphic to $H$, then $G$ is \\ nilpotent and its class is bounded by a function of three \\ variables: $H$, the exponent of $G$, and the soluble length of \\ $G$. It is a corollary that if the variety generated by a \\ soluble group $G$ of finite exponent contains $\stackrel{A^{2}}{\Rightarrow}$, then each \\ finite group in $\stackrel{A^{2}}{=}$ is isomorphic to some section of $G$.
}

In a recent paper [1], Macdonald and the first-named author proved (on the way to their Theorem 3.3) that the class of a metabelian regular $p$-group of exponent $p^{m}$ is at most $1+m(p-1)$. This paper is concerned with extending their result.

The first step (suggested by Dr James Wiegold) is to use a well-known argument based on a theorem of Hall [3] (sharpened by Stewart [6]) to eliminate the assumption "metabelian" at the cost of replacing the class bound $1+m(p-1)$ by a function which depends also on the soluble length of the group (and which we shall not attempt to optimize). The second is to note that while in [1] regular p-groups were nilpotent by definition, in this context it is sufficient to assume that we are dealing with a soluble group (of finite exponent) whose finite sections are all regular

Received 14 December 1970. 
p-groups. Thus if $\underline{\underline{H}}$ is the class of those finite $p$-groups which are not regular, then $\underline{\underline{H}}$ has the property $(p)$ : if $G$ is a soluble group of $p$-power exponent such that no section of $G$ belongs to $\underline{\underline{H}}$, then $G$ is nilpotent and its class is bounded in terms of its exponent and soluble length. It is then natural to ask what other classes of groups have $(p)$. (By a class of groups we always mean a union of isomorphism classes, but of course we do not insist that a class should contain the one-element groups.) The answer is that a class $\underline{\underline{H}}$ has $(p)$ if and (obviously) only if it contains at least one finite group from $\stackrel{A}{p}_{p}^{2}$ (the variety of elementary abelian by elementary abelian p-groups). Since the standard wreath product of two groups of order $p$ is an irregular group in $A_{p}^{2}$, this answer includes the claims made above. Its nontrivial core is the

THEOREM. If $H$ is a finite group in $\stackrel{\mathrm{A}^{2}}{\Rightarrow}$ and $G$ a soluble group of p-power exponent such that no section of $G$ is isomorphic to $H$, then $G$ is nilpotent and its class is bounded by a function of three variables: $H$, the exponent of $G$, and the soluble length of $G$.

The proof splits into two parts. The first, based on the Hall-Stewart Theorem mentioned earlier, results in

LEMMA 1. Let $G$ be a soluble group of length $s$ and exponent $p^{m}$. If the $\stackrel{A}{=}^{2}$-sections of $G$ are all nilpotent, then $G$ is nilpotent, and its class is bounded in terms of $s, p^{m}$, and the maximum of the classes of its $\stackrel{A}{=}_{p}^{2}$-sections.

The second part, which exploits properties of wreath products of elementary abelian $p$-groups and makes use of a result of Gupta and Newman [2], yields what is still needed for the Theorem:

LEMMA 2. Let $H$ be a finite group in $\stackrel{A}{p}^{2}$. If $G$ is a group in $\mathrm{A}^{2}$ such that no section of $G$ is isomorphic to $H$, then $G$ is nizpotent and its class is bounded in terms of $H$.

In fact, the result in [1] did not need to use the exponent of the 
group: the exponent of the commutator subgroup would have served equally well. Exploring the possibilities suggested by this fact, one may enquire about classes $\underline{\underline{H}}$ with the property $(p, c)$ : if $G$ is a soluble p-group such that the $(c+l)$ st term $\underline{N}_{C}(G)$ of the lower central series of $G$ has finite exponent and no section of $G$ belongs to $\underline{\underline{H}}$, then $G$ is nilpotent and its class is bounded in terms of $c$, the exponent of $\underline{N}_{c}(G)$, and the soluble length of $G$. The answer (for $c>0$ ) is that a class $\underline{H}$ has $(p, c)$ if and only if it contains at least one finite elementary abelian by cyclic $p$-group and at least one finite group from $\mathrm{A}_{\mathrm{p}}^{2}$. The proof runs along similar lines and we do not present it.

It is an immediate consequence of Lemma 2 that if a group $G$ generates $\stackrel{A^{2}}{p}$ then each finite group in $\stackrel{A^{2}}{\Rightarrow}$ is isomorphic to some section of $G$. We do not know of any other varieties with this property. For $\mathrm{A}^{2}$, we have the more general

COROLLARY. If a class $\underline{\underline{\mathrm{X}}}$ of groups generates a soluble variety of finite exponent which contains $\frac{\mathrm{A}^{2}}{p}$, then every finite group in $\frac{\mathrm{A}_{p}^{2}}{\Rightarrow}$ is isomorphic to some section of some group in $\underline{\underline{X}}$.

For, suppose $H$ is a finite group in $\stackrel{A^{2}}{\Rightarrow}$ which does not occur as a section of any group in $\underline{\underline{x}}$. By our Theorem, every p-section of every group in $\underline{X}$ is nilpotent of class at most $c$, say. By Lemma 4.3 of Higman [4], each finite group of $\frac{A^{2}}{p}$ is isomorphic to some section of some finite direct product $G_{1} \times \ldots \times G_{n}$ of groups from $\underline{\underline{X}}$, and hence to a section of a direct product $K$ of finite subgroups $K_{1}, \ldots, K_{n}$ of groups from $\underline{x}$. The $p$-sections of $K$ are sections of a sylow p-subgroup $P$ of $K$, and $P$ is isomorphic to a direct product of sylow p-subgroups $P_{i}$ of the groups $K_{i}$. As the $P_{i}$ are p-subgroups of groups in $\underline{\underline{x}}$, their classes do not exceed $c$, so the class of $P$ is also at most $c$. Thus it would follow that every finite group in $A^{2}$ p has class at most $c$, 
which is not the case.

Before proceeding to technicalities, we mention that it would be interesting to know more about classes, especially single isomorphism classes, which have the property $\left(p^{*}\right):$ if $G$ is a soluble group of $p$-power exponent such that no section of $G$ belongs to $\underline{\underline{H}}$, then $G$ is nilpotent. A slight extension of the proof of Lemma 2, together with Lemma 1 , shows that if $H$ is a direct product of a countably infinite elementary abelian $p$-group and a finite group from $\frac{A^{2}}{p}$, then (the isomorphism class of) $H$ has $\left(p^{*}\right)$. Does every extension of a finite elementary abelian $p$-group by a countable elementary abelian $p$-group have $\left(p^{*}\right)$ ? In particular, does a central product of countably many nonabelian groups of order $p^{3}$ have $\left(p^{*}\right)$ ? Does the countable, restricted direct power of a cyclic group of order $p^{2}$ have $\left(p^{*}\right)$ ? Can any nonnilpotent group have $\left(p^{*}\right)$ ? We have no answers.

The rest of the paper consists of the proofs of the two lemmas. We shall use the notation and terminology of Hanna Neumann's book [5], with three exceptions: we use "section" rather than "factor"; we write the verbal subgroup of a group $G$ corresponding to the variety $\underline{\underline{V}}$ as $\underline{\underline{V}}(G)$ rather than $V(G)$, and we do not reserve the letters $G$ and $H$ for relatively free groups. Some additional notation will be introduced as it becomes necessary.

Our first aim is to prove Lemma 1 under the additional assumption that $G \in \underline{\underline{A}} \stackrel{A}{\Rightarrow}$. This is done by induction on the exponent, say $p^{n}$, of $\stackrel{A}{\Rightarrow}(G)$. If $n=1$ then $G \in \frac{A^{2}}{\Rightarrow}$ and there is nothing to prove. For $n>1$, put $q=p^{n-1}$ and let $c$ denote the class of $G / \underline{A}_{q} \stackrel{A}{q}_{p}(G)$. If $g_{0}, g_{1}, \ldots, g_{2 c}$ are arbitrary elements of $G$, we have that $\left[g_{0}, g_{1}, \ldots, g_{c}\right]=g^{q}$ for some $g$ in $\underset{A_{p}}{(G)}$ and that $\left[g, g_{c+1}, \ldots, g_{2 c}\right] \in \underset{A}{A} \underset{P}{A}(G)$. Exploiting the facts that $G$ is metabelian and $\stackrel{A}{A}(G)$ has exponent dividing $q$, standard commutator calculations show that 


$$
\left[g_{0}, g_{1}, \ldots, g_{2 c}\right]=\left[g^{q}, g_{c+1}, \ldots, g_{2 c}\right]=\left[g, g_{c+1}, \ldots, g_{2 c}\right]^{q}=1
$$

so that $G$ is nilpotent of class (at most) $2 c$. (Clearly, a little more care would have produced a much better estimate, but we are only concerned here with showing the existence of a bound.)

We are now ready to prove Lemma 1 in general. Note that as $G$ is soluble of length $s$ and has exponent $p^{m}$, it lies in $\stackrel{A}{f s}_{p}^{m}$, so that if $k$ is the least positive integer with $G \in \underset{A^{k}}{\Rightarrow}$ then $k \leq m s$. The proof runs by induction on $k$, starting with trivial initial steps $k=1,2$. For the inductive step, put $N=\underline{A_{p}}(G)$ and assume, as $N \in \stackrel{A}{k-1}^{k-1}$, that $N$ is nilpotent and its class $d$ is bounded in terms of the relevant parameters. By the previous paragraph, we know that $G / N^{\prime}$ is also nilpotent and its class $c$ is similarly bounded. It remains to appeal to Stewart [6] to obtain that $G$ is nilpotent of class at most $c d+(c-1)(d-1)$. This completes the proof of Lemma 1 .

In preparation for the proof of Lemma 2, we note that it is sufficient to prove that lemma for the case when $H$ is a (standard) wreath product $W_{k}$ of a group $C$ of order $p$ and an elementary abelian group $c^{k}$ of order $p^{k}$. Indeed, suppose $H$ is any finite group in $\stackrel{A}{p}_{p}^{2}$, say, with $|\underline{\mathrm{A}}(H)|=p^{\alpha}$ and $|H / \stackrel{\mathrm{A}}{\Rightarrow} p(H)|=p^{\beta}$. By the embedding theorem (22.21 in [5]), $H$ can be embedded in $C^{\alpha}$ Wr $C^{\beta}$, which in turn (use 22.14 of [5]) can be embedded in $W_{k}$ provided $p^{k} \geq \alpha p^{\beta}$. Thus if no section of a group $G$ is isomorphic to $H$, no section of $G$ can be isomorphic to $W_{k}$ either.

As further preparation, we take the (presumably well-known) fact that $W_{k}$ is the unique extension of the regular $c^{k}$-module $v_{k}$ (over the field of $p$ elements: all modules considered will be over this field) by $c^{k}$. Uniqueness turns on the claim that every extension of $V_{k}$ by $c^{k}$ splits; 
one quick way to see this is by exploiting the details of the embedding theorem (22.21 of [5]) as follows. Let $V$ be an arbitrary extension of $V_{k}$ by $c^{k}$, and identify $V_{k}$ with the appropriate normal subgroup of $V$. Embed $V$ and $c^{k}$ in the wreath product $W$ of $V_{k}$ by $c^{k}$, the latter as a complement of the "base group" $B$ of $W$, in such a way that the action of $c^{k}$ on $V_{k}$ by conjugation in $W$ is the original regular action. Then $V_{k}$, as a regular and hence injective submodule of the $C^{k}$-module $B$, has a complement $K$ in $B$ which is also a submodule of $B$, that is, a normal subgroup of $W$. Since $V B=W$ and $V \cap B=V_{k}$ so that $V K=W$ and $V \cap K=1$,

$$
V \cong V K / K=W / K=c^{k} B / K=c^{k} K / K \cdot V_{k} K / K
$$

with $c^{k} K / K \cap V_{k} K / K=1$. As in the isomorphism above $V_{k}$ corresponds to $V_{k} K / K$, this shows that $V$ splits over $V_{k}$ as claimed.

We shall need one more fact, a direct consequence of the work of Gupta and Newman [2]: a group $G$ in $A^{2}$ is nilpotent of class $1+k(p-1)$ if $\left[g,(p-1) g_{1}, \ldots,(p-1) g_{k}\right]=1$ whenever $g \in G^{\prime}$ and $g_{1}, \ldots, g_{k} \in G$.

In order to prove Lemma 2, it is now sufficient to show that if $G$ is a group in $\stackrel{A}{2}_{p}^{2}$ and $g \in G^{\prime}, g_{1}, \ldots, g_{k} \in G$ such that $\left[g,(p-1) g_{1}, \ldots,(p-1) g_{k}\right] \neq 1$, then some section of $G$ is isomorphic to $W_{k}$. We proceed to select such a section. Let $A$ denote $A_{p}(G)$, and $S$ the subgroup of $G$ generated by $g_{1}, \ldots, g_{k}$, and $A:$ then $S / A$ is an elementary abelian $p$-group generated (possibly redundantly, for what we know at this stage) by $g_{1} A, \ldots, g_{k} A$, and $A$ is an $S / A$-module in the obvious sense. Denote by $U$ the normal closure of $g$ in $S$, that is, the submodule of $A$ generated by $g$. Write $W_{k}$ as a semidirect product $c^{k} V_{k}$; let $\left\{c_{1}, \ldots, c_{k}\right\}$ be a generating set of $c^{k}$, and $v$ a (free) 
generator of $V_{k}$ qua $C^{k}$-module. Consider $A$ also as a $C^{k}$-module via the homomorphism $\alpha$ of $c^{k}$ onto $S / A$ which maps each $c_{i}$ to $g_{i} A$, and let $\phi$ be the $C^{k}$-homomorphism of $V_{k}$ onto $U$ which maps $v$ to $g$. Now it is well known that $\left[v,(p-1) c_{1}, \ldots,(p-1) c_{k}\right]$ generates the unique minimal normal subgroup of $W_{k}$, that is, the unique minimal submodule of $V_{k}$; since this element is mapped by $\phi$ to the nontrivial element $\left[g,(p-1) g_{1}, \ldots,(p-1) g_{k}\right], \phi$ must be an isomorphism. As $V_{k}$ is a faithful $C^{k}$-module, this implies that so are $U$ and $A$, and therefore that $\alpha$ is also an isomorphism. Moreover, now we know that $U$ is a regular and hence injective submodule of $A$, so that $U$ has a complement $T$ in $A$ which is also a submodule, that is, a normal subgroup of $S$. It follows that $S / T$ is an extension of $A / T$ by $S / A$, or of $V_{k}$ by $C^{k}$ on account of $\alpha$ and the composite of $\phi$ with the natural isomorphism $U \rightarrow U T / T=A / T$. Hence $S / T \cong W_{k}$, and the proof is complete.

\section{References}

[1] Warren Brisley and I.D. Macdonald, "Two classes of metabelian p-groups", Math. 2. 112 (1969), 5-12.

[2] N.D. Gupta and M.F. Newman, "On metabelian groups", J. Austral. Math. Soc. 6 (1966), 362-368.

[3] P. Hall, "Some sufficient conditions for a group to be nilpotent", IZZinois J. Math. 2 (1958), 787-801.

[4] Graham Higman, "Some remarks on varieties of groups", Quart. J. Math. Oxford (2) 10 (1959), 165-178.

[5] Hanna Neumann, Varieties of groups (Ergebnisse der Mathematik und ihrer Grenzgebiete, Band 37, Springer-Verlag, Berlin, Heidelberg, New York, 1967). 
[6] A.G.R. Stewart, "On the class of certain nilpotent groups", Proc. Roy. Soc. Ser. A 292 (1966), 374-379.

University of Newcastle,

Newcastle,

New South Wales,

and

Institute of Advanced Studies,

Australian National University,

Canberra, ACT. 